\title{
A case of anomalous origin of the left coronary artery presenting with acute myocardial infarction and cardiovascular collapse
}

\author{
Twalib O Aliku ${ }^{1,2}$, Sulaiman Lubega ${ }^{2}$, Peter Lwabi²
}

1. Department of Paediatrics and Child Health, Gulu University.

2. Uganda Heart Institute.

\begin{abstract}
: week.

\section{Introduction}

Anomalous origin of the left coronary artery from the pulmonary artery (ALCAPA) is a common cause of myocardial infarction in children. ALCAPA typically presents in infancy with heart failure resulting from impaired left ventricular function that is often associated with mitral regurgitation(1). Myocardial ischemia arises as a result of decreased perfusion of the left ventricle with desaturated blood as a result of declining pulmonary artery pressures secondary to the decline in pulmonary vascular resistance that occurs normally after birth(1). This is worsened by the development of coronary steal phenomenon in which blood flows antegrade from the high pressure right coronary artery circulation, the collateral blood vessels and then retrograde through
\end{abstract}

Background: Though a rare clinical entity, anomalous origin of the left coronary artery from the pulmonary artery (ALCAPA) a common cause of myocardial infarction in children. Unrecognized and untreated it leads to progressive left ventricular dilatation and systolic dysfunction. In settings of high infectious burden, ALCAPA may erroneously be diagnosed as myocarditis, dilated cardiomyopathy or other common childhood disorders.

Clinical case: We present the case of a 10 weeks old male infant who presented to the inpatient unit with marked restlessness and irritability. He was inconsolable, had marked respiratory distress, cool extremities, central and peripheral cyanosis oxygen. The radial and brachial pulses were absent. The mean arterial pressure was $65 \mathrm{mmHg}$, Heart rate of 160 beats per minute with a third heart sound. The liver was enlarged $4 \mathrm{~cm}$ below the costal margin and tender, with a splenomegaly. He had an elevated Creatinine Kinase-MB of $112.5 \mathrm{u} / \mathrm{L}$. ECG revealed deep Q waves in leads I, aVL, V5, V6 with ST elevation in the anterolateral leads. Echo showed a dilated left ventricle LVEDd of $40 \mathrm{~mm}$, with paradoxical interventricular septal motion, severe LV systolic dysfunction ( $\mathrm{FS}=15 \%, \mathrm{EF}=28 \%$ ), $\mathrm{LV}$ anterolateral wall echo brightness and flow reversal in the Left coronary artery with its origin from the pulmonary trunk. He was admitted to the coronary care unit as a case of acute myocardial infarction with cardiovascular collapse. He received fluid resuscitation, inotropic support and standard management of heart failure. Six days later he was discharged home with a plan to refer abroad. He died at home after one

Conclusion: A combination of a high index of suspicion, typical ECG and echocardiographic findings in a young infant presenting with LV dysfunction could lead to an earlier diagnosis of ALCAPA.

African Health Sciences 2014;14(1): 223-227 http://dx.doi.org/10.4314/ahs.v14i1.35

\section{Corresponding author: \\ Twalib Olega Aliku \\ Department of Paediatrics and Child Health \\ Faculty of Medicine, Gulu University \\ P.O Box 166, Gulu Uganda \\ Email: aliku90@yahoo.com}

the anomalous left coronary artery to the lower pressure pulmonary trunk(2). Many common childhood disorders in the tropics may mimic this anomaly. We present the case of an infant diagnosed with ALCAPA presenting with acute myocardial infarction and cardiovascular collapse initially misdiagnosed as dilated cardiomyopathy secondary to myocarditis.

\section{Case report}

We present the case of a 10 weeks old male infant who had been seen a month earlier in our cardiology service with fever, cough and difficulty in breathing. His echo showed dilated left ventricle with poor systolic function that was attributed to myocarditis or dilated cardiomyopathy. Four weeks later he presented to the inpatient unit with marked restlessness and irritability. He had no cough, fever, coryza, edema, diarrhea or vomiting. He was inconsolable, had marked respiratory distress, cool extremities, central and peripheral cyanosis oxygen saturations of $45-57 \%$ in room air. The radial and brachial pulses were absent. The mean arterial pressure was $65 \mathrm{mmHg}$, Heart rate of 160 beats per minute with a third heart sound. The liver was enlarged $4 \mathrm{~cm}$ below the costal margin and tender, with a splenomegaly. The 
respiratory exam revealed severe subcostal retractions with bronchovesicular breath sounds and no added sounds $\mathrm{He}$ had an elevated Creatinine Kinase-MB of $112.5 \mathrm{u} / \mathrm{L}$. His total white cell count was 16800/ micromil, $61.9 \%$ lymphocytes and a Hemoglobin of $10.4 \mathrm{~g} / \mathrm{dl}$. Serum sodium was $133.7 \mathrm{mmol} / 1$, the potassium $4.9 \mathrm{mmol} / 1$. The serum urea was $4.7 \mathrm{mmol} / 1$ and creatinine of $40 \mathrm{micromol} / \mathrm{ml}$. The chest radiograph revealed a cardiomegaly. ECG revealed deep Q waves in leads I, aVL, V5, V6 with ST elevation in the anterolateral leads see figure 1. Echo showed a dilated left ventricle LVEDd of $40 \mathrm{~mm}$, with paradoxical interventricular septal motion, severe LV systolic dysfunction ( $\mathrm{FS}=15 \%, \mathrm{EF}=28 \%$ ), $\mathrm{LV}$ anterolateral wall echo brightness and flow reversal in the Left coronary artery with its origin from the pulmonary trunk. He was admitted to the coronary care unit as a case of acute myocardial infarction with cardiovascular collapse. He received fluid resuscitation, inotropic support with low dose dopamine at 5 microgram $/ \mathrm{kg} / \mathrm{min}$ for two days, and standard management of heart failure including captopril, spironolactone, carvedilol, frusemide and digoxin. The dopamine was discontinued 2 days later. Six days later he was clinically improved with resolution of respiratory distress, oxygen saturations of $98 \%$ in room air, with normal peripheral perfusion, BP was $88 / 50 \mathrm{mmHg}$ and he was discharged. At the time of discharge the ST segments had returned to baseline and the repeat Creatinine Kinase-MB was $25 \mathrm{mmol} / \mathrm{ml}$. (See figure 2).

He died at home with no postmortem performed one week after hospital discharge while awaiting referral abroad since the Uganda Heart Institute still lacks capacity to operate on these cases.

Figure 1: ECG at the time of admission. Note the ST segment elevation in the anterolateral leads

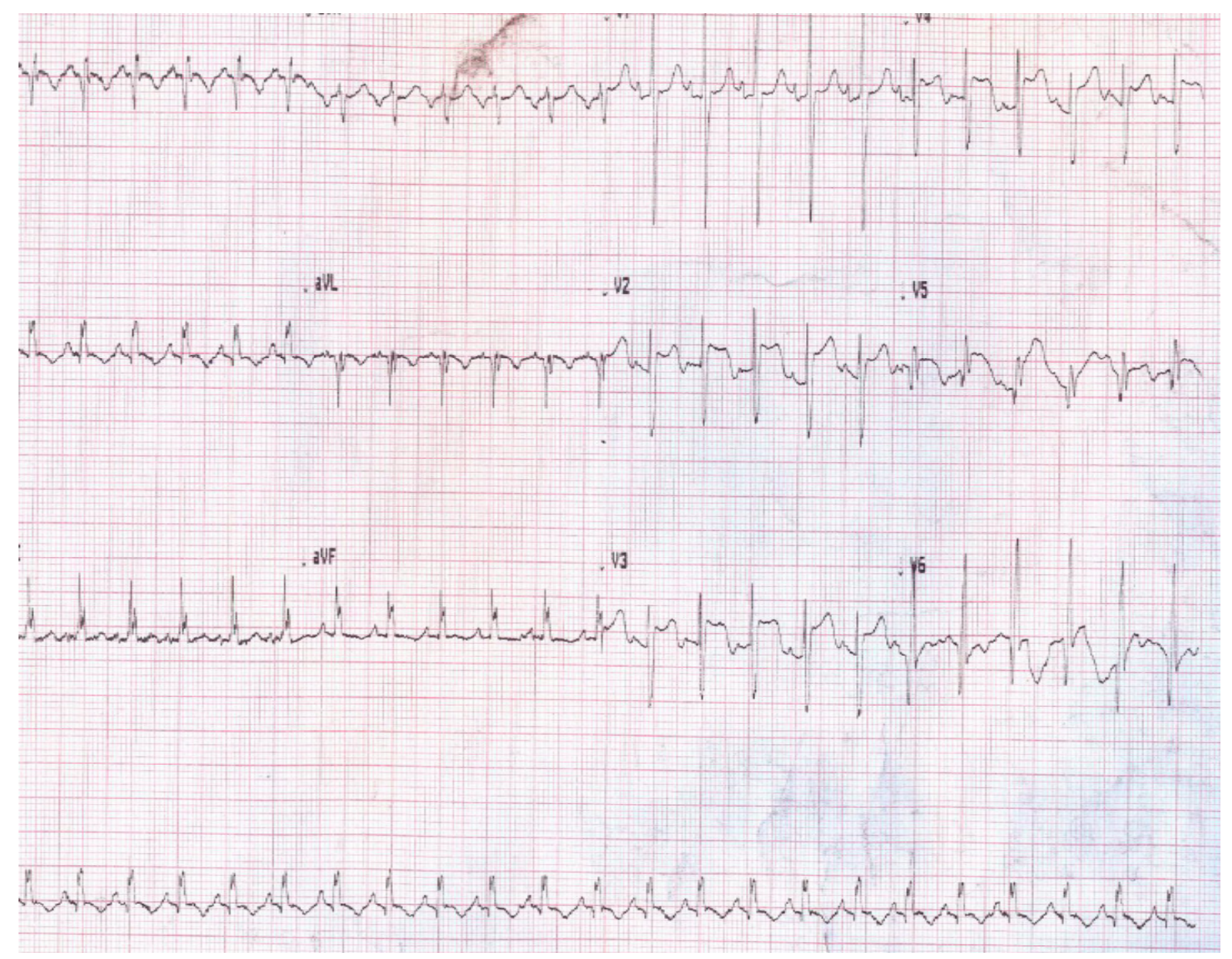


Figure 2 Acase of anomalous origin of LCAPA

Figure 2: ECG of the patient at discharge. The ST segment elevation in V2-V5 has returned to baseline, with the persistence of $\mathrm{Q}$ waves in leads I and aVL.

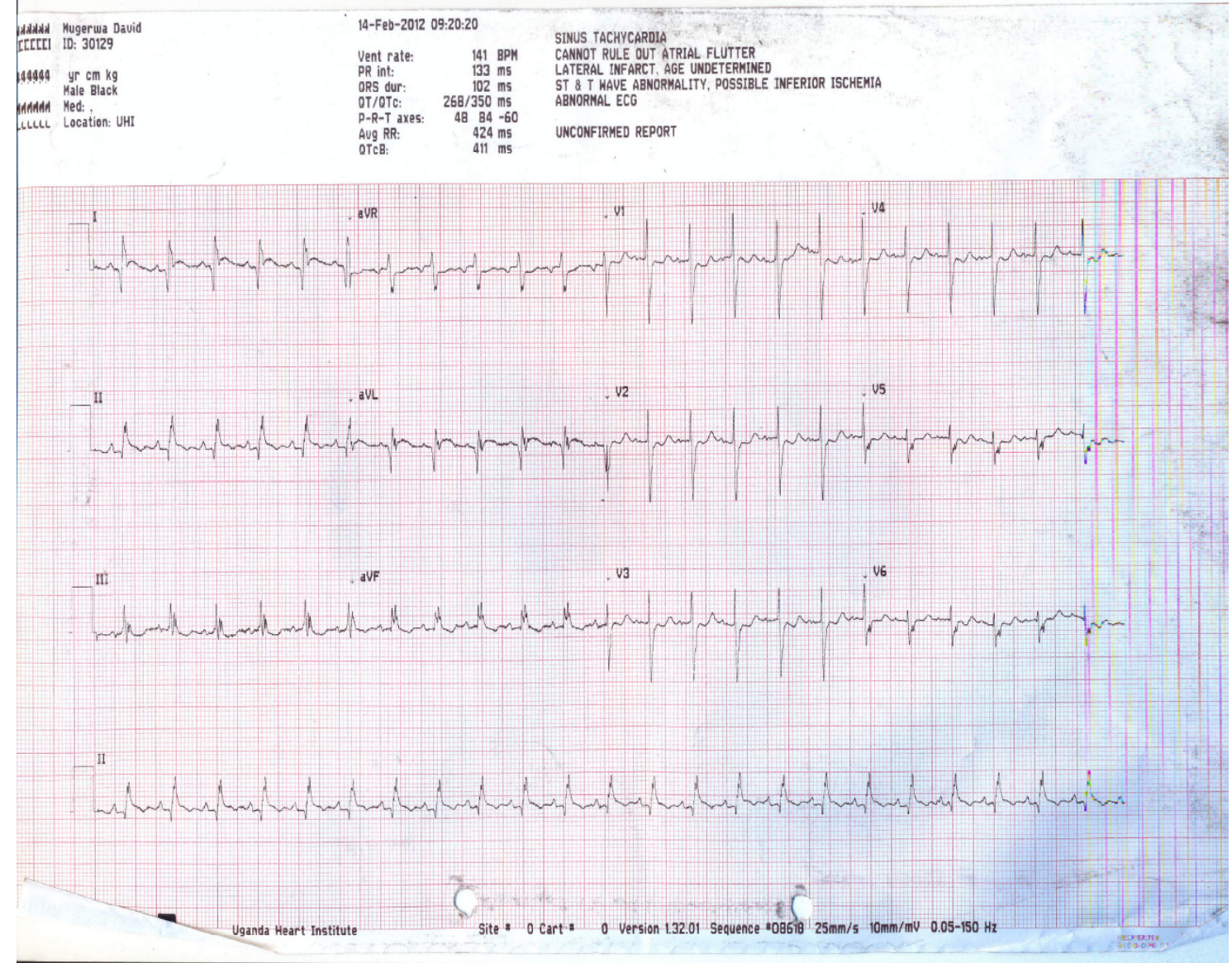

Figure 3: Echocardiagraphic features of ALCAPA in a 6 month old Infant

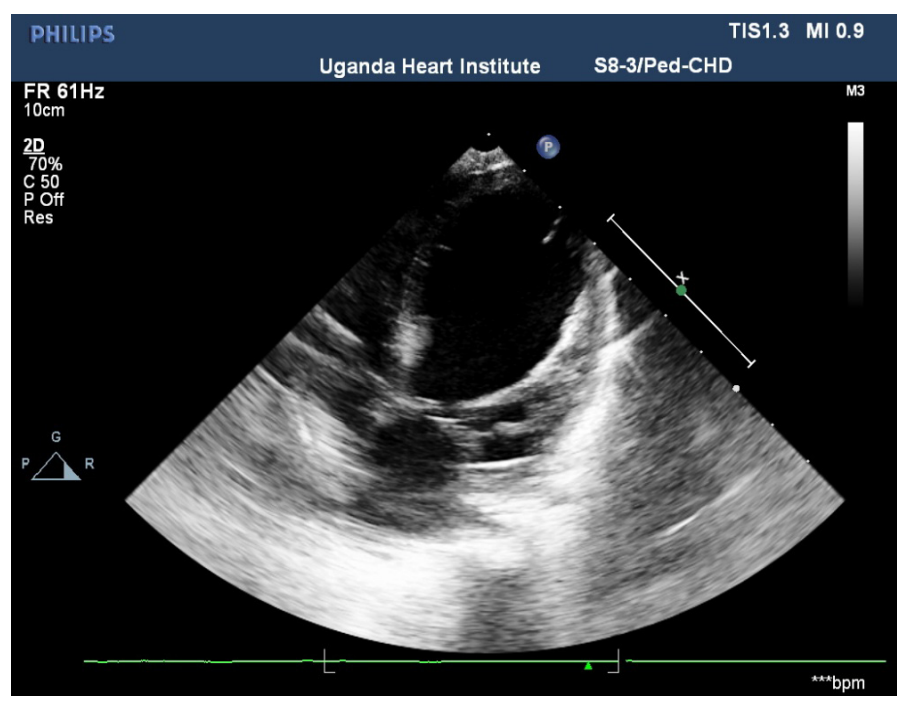

Figure 3a:Apical 4 chamber view . Note the LV lateral wall endocardial echobrightness from endocardial ischaemia on the left panel (arrow). The left ventricle is was dilated with moderate $\mathrm{LV}$ dysfunction in this

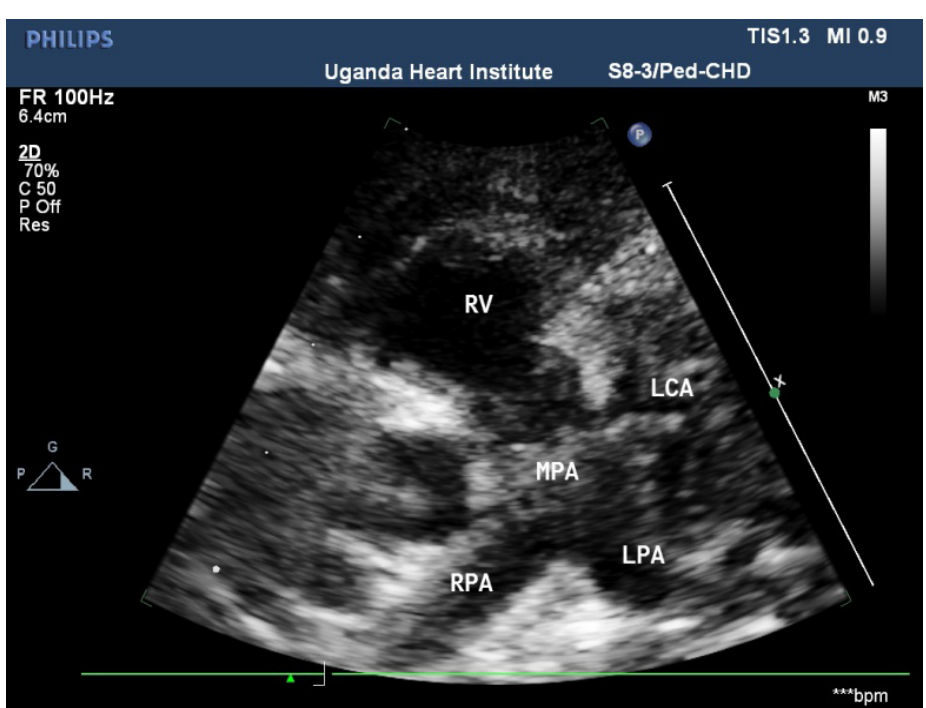

patient. The Left coronary artery LCA arises directly from the MPA (main pulmonary artery trunk) in the panel on the right 
Figure 3b: High Parasternal short axis view showing a reversed flow pattern through the Anomalous left coronary artery. MPA: Main pulmonary artey. RPA: Right pulmonary artery. LPA: Left pulmonary artry. RV: Right ventricle

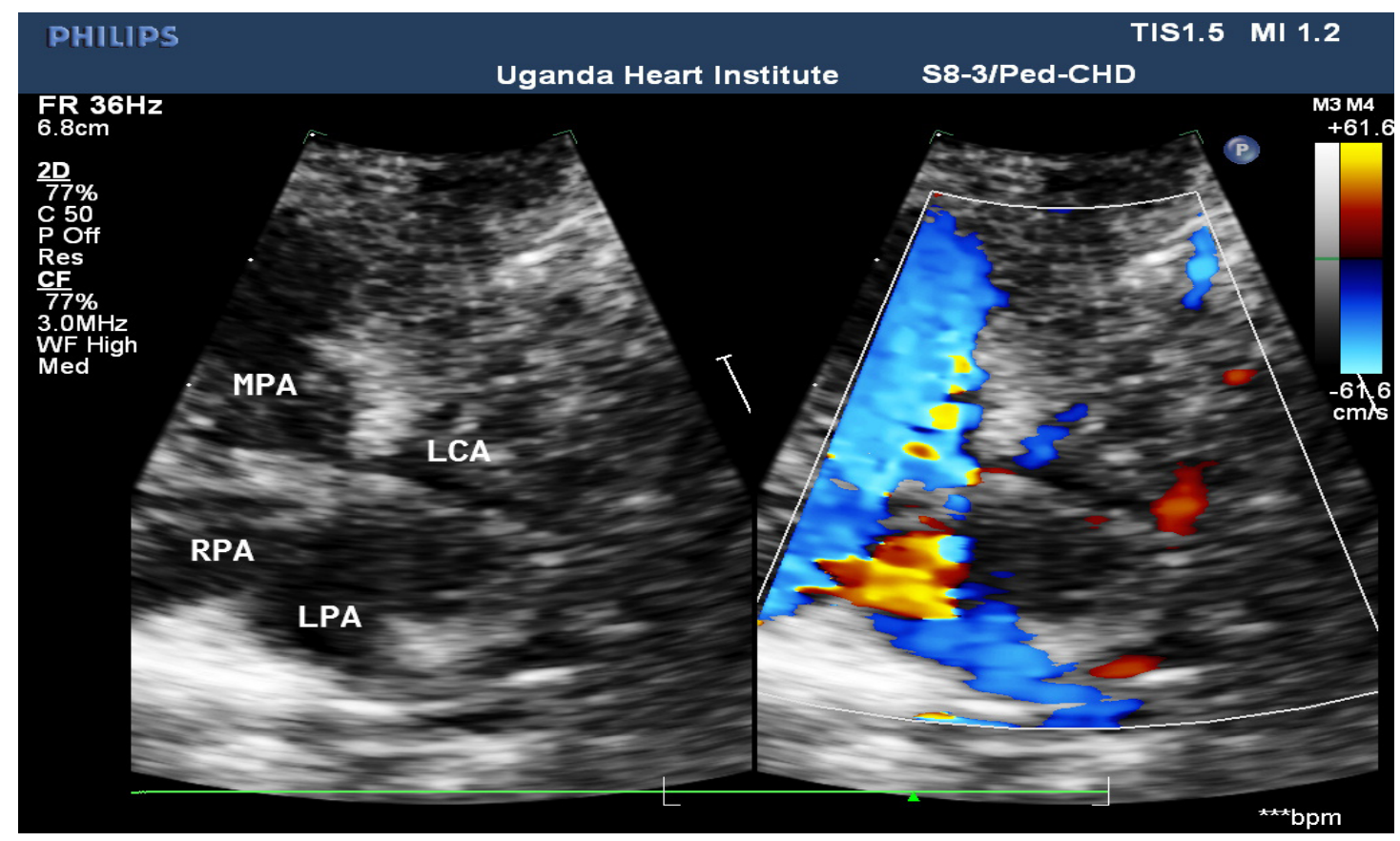

\section{Discussion:}

Anomalous origin of the left coronary artery from the pulmonary artery (ALCAPA) is a common cause of myocardial infarction in children. Because it is a rare disorder, occurring in about $0.023 \%$ of live births (2), it is often missed. Unrecognized and untreated it eventually leads to progressive LV dilatation, systolic dysfunction and congestive heart failure that is often made worse by mitral regurgiataion secondary to a dilated mitral ring or infarction and dysfunction of the anterolateral papillary muscle(1). The paroxysms of marked restlessness and irritability that is characteristic, as shown in this case, correlating with episodes of angina pectoris is a common presenting feature in early infancy. These are often triggered by the exertion of nursing or crying and may be erroneously attributed to relatively common disorders such as infantile colic, food intolerance, garstroesophageal reflux(2) or dehydration from diarrhea. Indeed if this patient had diarrhea or vomiting, in a tropical setting this would be attributed to dehydration and so missing the diagnosis. The absence of diarrhea or vomiting with marked irritability and cardiovascular collapse was a clue to evaluate the patient further. The presence of fever and cough at the initial echocardiographic examination caused an erroneous attribution of the severe left ventricular dysfunction to myocarditis. If a 12 lead ECG had been done at the initial evaluation, possibly the diagnosis of ALCAPA could have been made earlier.

Early recognition of this rare anomaly and differentiating it from other causes of dilated cardiomyopathy is important for early surgical correction so as to prevent the development of extensive myocardial damage. The ECG diagnosis is often easy, since ALCAPA commonly presents with myocardial infarction in infancy but the echocardiographic diagnosis may be challenging. Even though left ventricular dysfunction is very common in infants with ALCAPA and helps to increase the index of suspicion during echocardiography, rarely patients may present with normal left ventricular function(3). Normal Left ventricular function would occur in the setting of pulmonary arterial hypertension that permits forward flow in the left coronary artery or those with rapid development of adequate collaterals between the right and left coronary circulations(3). Echocardiographic features predictive of ALCAPA include regional wall motion abnormalities in an infant with dilated LV with systolic dysfunction, LV anterolateral echo brightness 
from myocardial ischemia(3-4), dilated right coronary artery with concordant flow pattern (4-5), coronary artery arising from the pulmonary trunk and flow reversal in the left coronary artery(1).

Our patient died one week after hospital discharge while awaiting referral abroad for corrective surgery. Without surgical therapy, up to $90 \%$ of children die in infancy(6). The few patients with ALCAPA that survive to childhood may present with heart failure(4), chest pain(5), syncope or sudden cardiac death from ventricular arrhythmias(7).

\section{Conclusion}

ALCAPA may be missed in an infant with heart failure and having LV dilatation with systolic dysfunction. However with a high index of suspicion, typical ECG and Echo features the diagnosis of a potentially treatable cause of dilated cardiomyopathy should be easy to make.

\section{Acknowledgements}

Special thanks to Dr Craig Sable for the echo teaching on echocardiographic features of ALCAPA and Dr Charles Berul for the electrocardiographic findings in ALCAPA

\section{References}

1. Marthene P G, Lim S D. Congenital anomalies of the coronary vessels and aortic root. Moss and Adams heart disease in infants, children and adolescents including the fetus and young adult. 7th ed (vol 1). Lippincott Williams and Wilkins. 2008

2. Brotherton H, Phillip RK. Anomalous origin of left coronary artery from the pulmonary artery (ALCAPA) in infants: A five year review in a defined birth cohort. Eur J Pediatr. 2008;167:43-6.

3. Kurup R P, Daniel R, Kumar R K. Anomalous origin of the left coronary artery from the pulmonary artery in infancy with preserved left ventricular function: potential pitfalls and clues to diagnosis. Ann Pediatr Cardiol 2008;1(1):65-7.

4. Grimaldi A, Ammirati E, La Canna G, Sora N , et al. Echocardiographic 'brainstorm' to detect anomalous origin of the left coronary artery from the pulmonary artery". Journal of cardiovascular medicine. 2012;13:152-155

5. Koike K, Musewe N N, Smallhorn J F, Freedom R M. Differentiating between anomalous origin of the left coronary artery from the pulmonary trunk and dilated cardiomyopathy: role of echocardiographic measurement of the right coronary artery Br Heart J 1989;61:192-7.

6. Artman M, Mahony C, Teitel D F. Neonatal cardiology.2nd ed. McGraw-Hill, New York. 2010

7. Neilsen, HB, Perko M, Aldershivile J, Saunamaki K. Cardiac arrest during exercise: Anomalous origin of the left coronary artery from the pulmonary trunk. Scand Cardiovasc J 1999;33:369-71. 